\title{
Space representation of stochastic processes with delay
}

\author{
Silvio R. Dahmen, ${ }^{1,2}$ Haye Hinrichsen, ${ }^{1}$ and Wolfgang Kinzel ${ }^{1}$ \\ ${ }^{1}$ Fakultät für Physik und Astronomie, Universität Würzburg, Am Hubland, D-97074 Würzburg, Germany \\ ${ }^{2}$ Instituto de Física da UFRGS, Avenida Bento Gonçalves 9500, 91501-970 Porto Alegre, Brazil
}

(Received 28 March 2007; revised manuscript received 24 July 2007; published 6 March 2008)

\begin{abstract}
We show that a time series $x_{t}$ evolving by a nonlocal update rule $x_{t}=f\left(x_{t-n}, x_{t-k}\right)$ with two different delays $k<n$ can be mapped onto a local process in two dimensions with special time-delayed boundary conditions, provided that $n$ and $k$ are coprime. For certain stochastic update rules exhibiting a nonequilibrium phase transition, this mapping implies that the critical behavior does not depend on the short delay $k$. In these cases, the autocorrelation function of the time series is related to the critical properties of the corresponding twodimensional model.
\end{abstract}

DOI: 10.1103/PhysRevE.77.031106

PACS number(s): 05.40.-a, 64.60.Ht, 05.45.Tp

\section{INTRODUCTION}

Dynamical systems with time-delayed feedback show interesting phenomena and have attracted considerable attention due to their relevance in different research fields: nonlinear dynamics [1], neurobiology [2], chaos control [3], synchronization [4,5], and communication [6]. In particular, in the context of laser physics [7], recent progress on a public-channel cryptography system based on the synchronization of chaotic lasers [8] has motivated the authors to better understand the physics of time-delayed differential equations, a subject less explored and not as well understood as the physics of ordinary differential equations [9]. Most of the literature on the subject of delayed feedback concentrates on deterministic systems [10]. However, stochastic systems with time-delayed feedback are not so well understood [11] and may play an important role as, for example, in the context of gene regulation [12]. Moreover, for such systems the evolution is often discrete in time.

As a step toward a better general understanding of timedelayed systems we investigate in this paper a simple discrete model with delay: a stochastic process for a single binary variable which evolves according to its own history. We show that this model can be mapped onto a two-dimensional stochastic cellular automaton in such a way that the timedelayed couplings become local. As a result, the delay becomes a boundary effect and, if one chooses the evolution laws properly, the autocorrelation function of the corresponding time series can be related to the critical properties of the cellular automaton, in our example a directed percolation process. The results are, however, more general and may be applied to a large variety of systems.

The general idea of the mapping of a time series onto a $(1+1)$-dimensional system was already introduced by Giacomelli and Politi in the context of deterministic differential equations with a single delay [13]. Here we generalize this concept to discrete stochastic systems with two delays. Moreover, we show that, for update rules that correspond to a critical system in two dimensions, the critical exponents, which are usually hidden in a scrambled data set, can be extracted through an appropriate reordering of the time series.

\section{REORDERING OF THE TIME SERIES}

We consider a time series $x_{t}$ with a discrete time variable $t \in \mathbb{N}$ that evolves by nonlocal stochastic updates $\left(x_{t-n}, x_{t-k}\right)$ $\rightarrow x_{t}$ in such a way that the probability for the outcome $x_{t}$ is given by

$$
P\left(x_{t}\right)=f\left(x_{t}, x_{t-n}, x_{t-k}\right), t>n>k,
$$

where $n$ and $k$ are two different delays. The initial configuration may be given by specifying $n$ subsequent elements of the time series, e.g., $x_{0}, \ldots, x_{n-1}$. The type of data represented by $x_{t}$ and the function $f$ are not restricted in any way; the only important ingredient is that a new entry of the time series $x_{t}$ depends on previous values of $x$ at times $t-n$ and $t-k$.

The main result of this paper can be summarized as follows: irrespective of the structure of $x_{t}$ and $f$, it is possible to rearrange the time series in such a way that the couplings become local in a two-dimensional representation. More specifically, it is possible to define a reordered series $y_{t}$ that evolves by updates $\left(y_{t-n}, y_{t-s}\right) \rightarrow y_{t}$ with

$$
P\left(y_{t}\right)=f\left(y_{t}, y_{t-n}, y_{t-s}\right),
$$

where $s$ is a different delay that takes the values 1 or $n+1$ in a precisely defined mathematical way (see the Appendix for details). The only condition for this transformation to work is that the original delays $n$ and $k$ have to be coprime, i.e., have no common divisor other than 1 .

Before discussing the mathematical details of this transformation and the coprimality condition, we present, for the sake of clarity, a geometrical construct which explains how the transformation works for two special values of $n$ and $k$.

Consider an arbitrary time series with delays $n=7$ and $k$ $=3$. One starts out with a given configuration $\left\{x_{0}, x_{1}, \ldots, x_{6}\right\}$ and, from this initial condition, constructs a time series by iteration of Eq. (1). This time line is represented in the upper part of Fig. 1 where arrows indicate which terms are causally connected. The lower part of the same figure depicts what the causal connection would look like after reordering, as explained below.

The transformation can be seen as a sequence of three steps. The first one is the stacking up of different blocks of size $n$. Following Giacomelli and Politi [13], the time series 

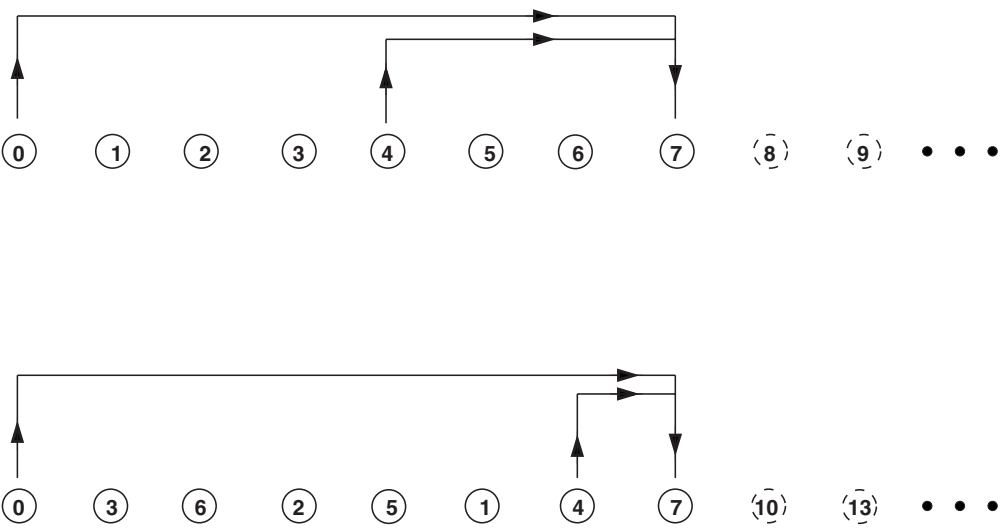

FIG. 1. Updating scheme in systems with delays $n=7$ and $k=3$ before (upper figure) and after (lower figure) the transformation (6). The arrows indicate the couplings needed to update the variable $x_{7}$ as a function of $x_{0}$ and $x_{4}$.

is divided into equidistant segments of $n$ elements which are arranged line by line on top of each other. This means that the index $t$ is mapped to the position

$$
\left(\begin{array}{l}
x \\
y
\end{array}\right)=\left(\begin{array}{c}
t \bmod n \\
\lfloor t\rfloor_{n}
\end{array}\right) .
$$

When the original time series $x_{t}$ is drawn in such a $(1+1)$-dimensional representation, the long delay $n$ turns into a nearest-neighbor interaction in the vertical direction while the short delay is still nonlocal (see Fig. 2).

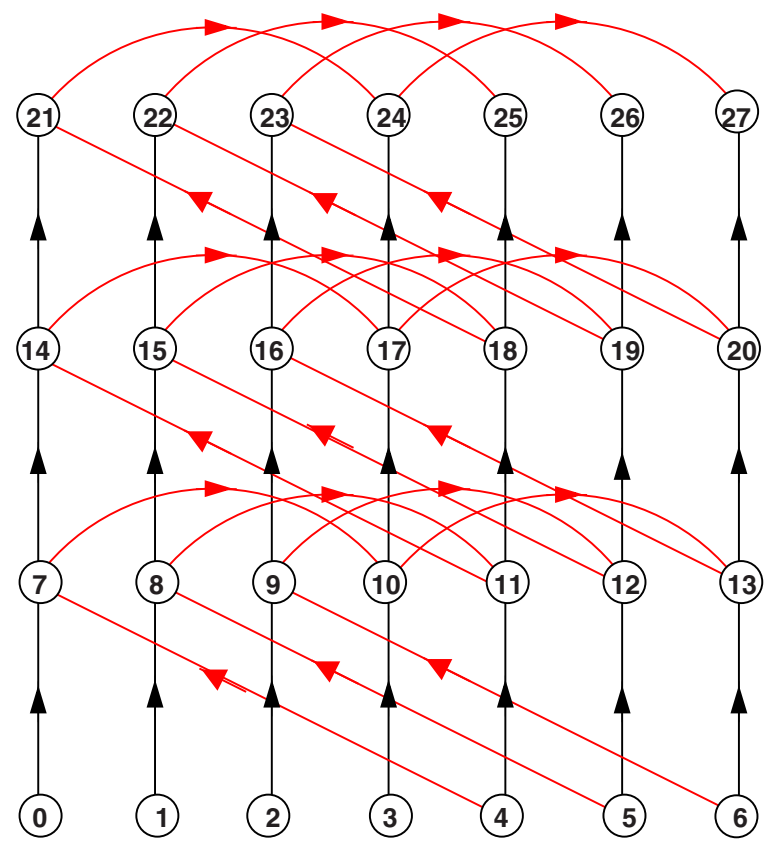

FIG. 2. (Color online) Original time series $x_{t}$ with delays $n=7$ and $k=3$ in a two-dimensional representation [13]. The time series is divided into segments of size $n$ which are plotted line by line on top of each other. Starting with a given configuration $\left\{x_{0}, x_{1}, \ldots, x_{6}\right\}$, the whole time series can be constructed by iteration of Eq. (1); here the black arrows represent the long delay between $x_{t-n}$ and $x_{t}$ while the red ones indicate the short delay between $x_{t-k}$ and $x_{t}$. The figure is equivalent to the upper part of Fig. 1, the only difference being that blocks of size 7 are arranged as a twodimensional grid.
The second step is the reordering (shuffling) of the time indices, a procedure applied to each separate block of size $n$ in such a way that they do not get mixed. For the particular case depicted in this section the first $n$ sites are reordered according to

$0 \rightarrow 0 ; 1 \rightarrow 3 ; 2 \rightarrow 6 ; 3 \rightarrow 2 ; \quad 4 \rightarrow 5 ; \quad 5 \rightarrow 1 ; 6 \rightarrow 4$

This should be read as: site 0 remains where it is; in the place where site 1 was one should write site 3 , and so on. The pairs are obtained via the operation (new position) $=($ old position $) \times 5 \bmod (7)$. The same reordering scheme takes place in the subsequent blocks. As illustrated in Fig. 3, this transformation preserves the long delay $n$ while the short delay $k$ is mapped onto 1 or $n+1$ according to Eq. (A4). As we will see, this also holds in the general case as long as $n$ and $k$ are coprime. Note that the corresponding boundary conditions are not periodic but shifted in the vertical direction, connecting subsequent blocks periodically in a spirallike manner.

Although the couplings in Fig. 3 are local, they are still biased toward the north-east. Moreover, the coupling scheme exhibits vertical dislocation lines. So, in order to remove these irregularities, one may redraw the figure in such a way that all updates have the same orientation in the $x y$ plane as illustrated in Fig. 4. This is the third and final step of the transformation, and allows one to relate the original time series to $(1+1)$-dimensional cellular automata on a tilted square lattice. However, by rearranging the lattice one ob-

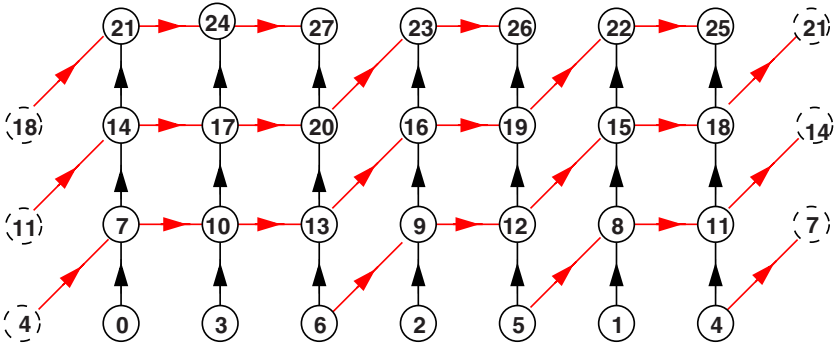

FIG. 3. (Color online) Corresponding reordered time series in a two-dimensional representation according to Eq. (6), where the bulk interactions become local. 


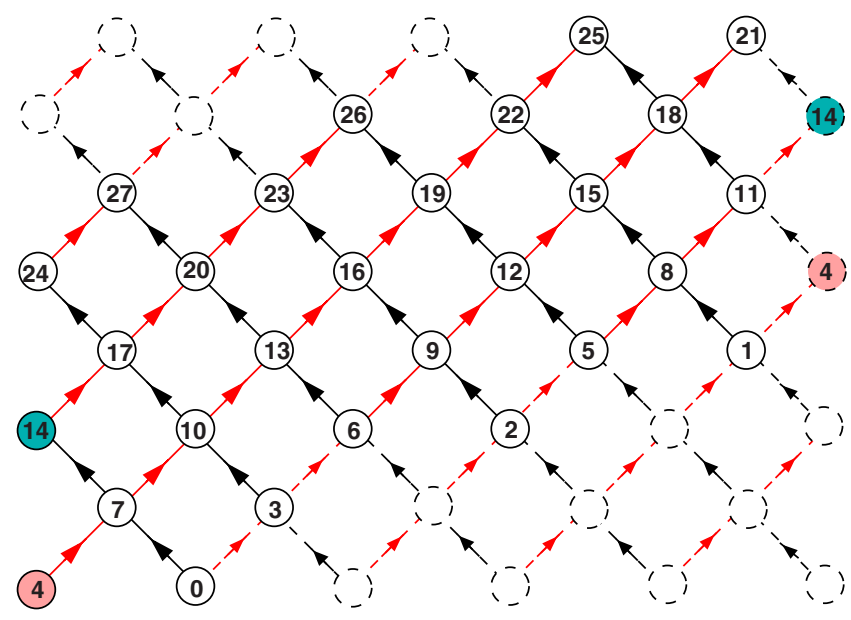

FIG. 4. (Color online) The same lattice as in Fig. 3 plotted in such a way that all updates in the bulk are geometrically identical. The boundary conditions one obtains can be seen as being time delayed (time flows upward).

tains skewed boundary conditions with a nonlocal delay connecting blocks that are vertical distance $n-k$ apart.

\section{MATHEMATICAL FORMULATION}

In this section we present the rigorous mathematical formulation of the mapping introduced in the previous section. The transformation from a one-dimensional to a $(1+1)$-dimensional form is rather straightforward (steps 1 and 3 in the last section). We therefore concentrate on the reordering (shuffling) of the time series, which makes the short delay $k$ local while keeping $n$ unchanged. Starting with the updating rule Eq. (1), the reordered time series $y_{t}$ of Eq. (2) is related to the original one by

$$
x_{t}=y_{T(t)},
$$

where the map $T(t)$ is given by

$$
T(t)=(t q) \bmod n+n\lfloor t\rfloor_{n} .
$$

In this equation $\lfloor t\rfloor_{n}$ denotes integer division by $n$ while $q$ $\in\{0, \ldots, n-1\}$ is an integer such that

$$
(k q) \bmod n=1 .
$$

The existence of an integer $q$ satisfying the equation above guarantees that the mapping is one to one and has the inverse

$$
T^{-1}(t)=(t k) \bmod n+n\lfloor t\rfloor_{n} .
$$

The meaning of the mapping (6), which we prove rigorously in the Appendix, is simple: the first term on the right-hand side (RHS) reorders the indices, while the second term places them into separate blocks of size $n$.

In mathematical parlance, Eq. (7) means that $n$ and $k$ are coprime. Coprimality, albeit necessary, is not restrictive in the sense that the number of coprimes to a given $n$, as given by Euler's totient function $\phi(n)$, is known to increase sufficiently rapidly with increasing $n$ (faster than $\sqrt{n}$ ). This means that in the large- $n$ limit the transformation can be applied to systems with various delays $k$. If the original delays $n$ and $k$ are not coprime, then the mapping is no longer injective. Moreover, if $n$ is a multiple of $k$ the original system breaks up into $\lfloor n\rfloor_{k}$ independent time series which evolve without mixing.

To demonstrate how the transformation works let us consider a simple deterministic update rule where $x_{t} \in 0,1$ is a binary time series. The update rule is given by the Boolean function

$$
x_{t}=x_{t-n} \oplus x_{t-k}= \begin{cases}1 & \text { if } x_{t-n} \neq x_{t-k}, \\ 0 & \text { otherwise }\end{cases}
$$

where $\oplus$ denotes a logical XOR operation. Such a binary time series can be visualized by plotting $x_{t}$ as black and white pixels at position $(x, y)$. The results are shown in Fig. 5, where we used the delays $n=300$ and $k=227(q=263)$. Starting with a single nonzero entry in the initial state $x_{n / 2}=1$, the iteration of the update rule (1) produces an irregular pattern of pixels, which is shown in the top panel of Fig. 5. When the transformation (6) is applied, the pixels are ordered with a bias to the north-east, resulting in a tilted Sierpinsky gasket (see middle panel). Finally, plotting the same data in such a way that the tilt is removed, one obtains the usual form of the Sierpinsky gasket (see bottom panel). However, as exemplified by the arrow, the boundary conditions are no longer periodic; instead they involve a nonlocal shift $\delta=n-k$ in the vertical direction.

\section{STOCHASTIC UPDATE RULE RELATED TO DIRECTED PERCOLATION}

Let us now turn to a simple but nontrivial example of a stochastic update rule. This example serves the purpose of illustrating how the critical behavior of a time series with delay manifests itself. For a binary time series a probabilistic update $\left(x_{t-n}, x_{t-k}\right) \rightarrow x_{t}$ according to Eq. (1) is determined by

$$
\begin{gathered}
f\left(1, x_{i}, x_{j}\right)=p_{x_{i}, x_{j}}, \\
f\left(0, x_{i}, x_{j}\right)=1-p_{x_{i}, x_{j}},
\end{gathered}
$$

with four control paramters $p_{00}, p_{01}, p_{10}, p_{11}$. In what follows let us assume that $p_{00}=0$. In this case the time series consisting of zeros is a fixed point of the dynamics. In nonequilibrium statistical physics, such a configuration, which can be reached but not left, is called an absorbing state. Whether or not this absorbing state is stable against perturbations depends on the magnitude of the remaining control parameter $p_{01}, p_{10}$, and $p_{11}$. For example, using an update rule inspired by the Domany-Kinzel (DK) model, we have

$$
p_{01}=p_{10}=p, \quad p_{11}=2 p-p^{2} .
$$

In the corresponding time series, on varying $p$ between 0 and 1 one indeed observes the following phenomenological behavior.

(1) If $p$ is very small the time series quickly approaches the absorbing series consisting of zeros. 

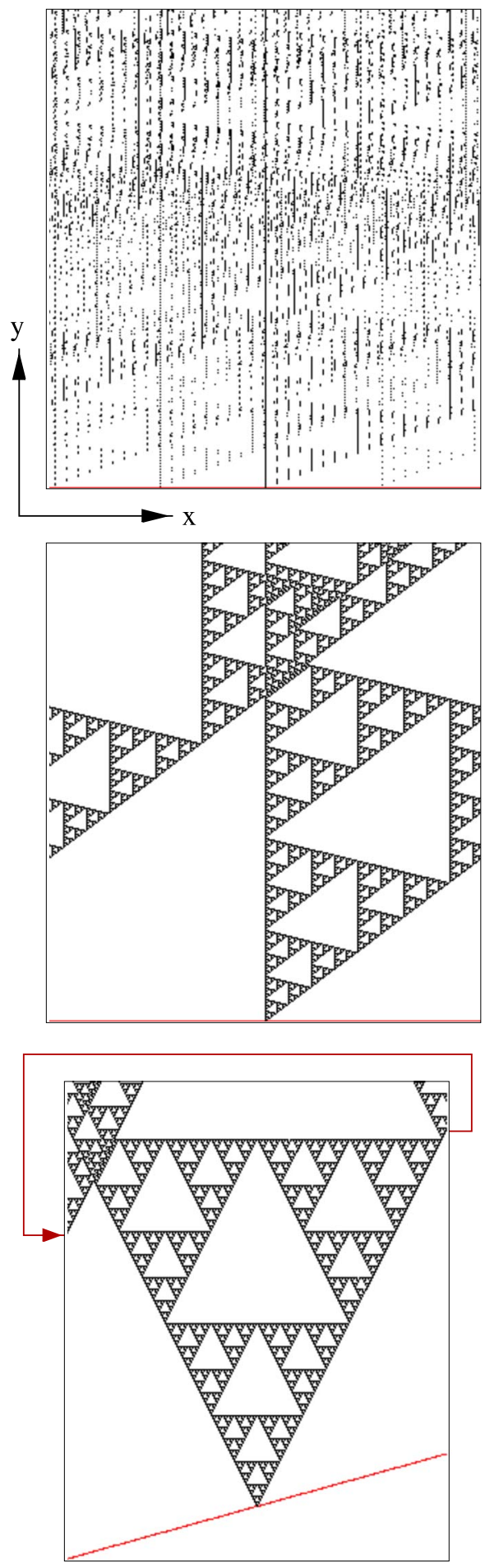

FIG. 5. (Color online) Visual representation of the time series generated by the update rule (9). The three panels correspond to the examples shown above in Figs. 2-4 (see text).

(2) For large $p$ the dynamics approaches a fluctuating steady state with a nonvanshing stationary expectation value of $x_{t}$. The probability of reaching the absorbing configuration is very low and decreases with increasing $n$.

(3) At a certain threshold $p_{c} \approx 0.6447$, one observes a power-law decay of the density in a finite temporal range which grows with $n$.
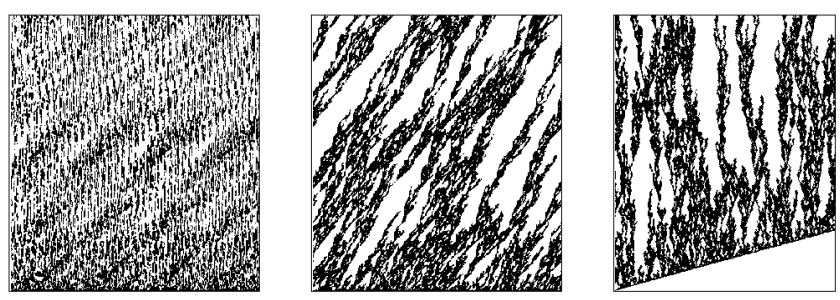

FIG. 6. Analogous representation of a stochastic time series with $n=300$ and $k=227$ evolving by a directed percolation update rule at the critical point $p=p_{c}$. Here the iteration starts with a sequence $111 \ldots$.

Since the DK model belongs to the universality class of directed percolation (DP) [15-18], it is natural to expect the system to display a precursor of DP critical behavior. In fact, using the transformation (6), the update rule becomes equivalent to that of a Domany-Kinzel cellular automaton [14]. Using the present notation the $(1+1)$-dimensional Domany-Kinzel model is defined on a tilted square lattice with coordinates $(x, y)$. Each lattice site can be either active $[s(x, y)=1]$ or inactive $[s(x, y)=0]$. The model evolves by parallel updates, i.e., the new horizontal line at $y+1$ is obtained by setting

$$
s(x, y+1):= \begin{cases}1 & \text { with probability } p_{s(x-1, y), s(x+1, y)}, \\ 0 & \text { otherwise }\end{cases}
$$

For the choice $p_{01}=p_{10}=p$ and $p_{11}=2 p-p^{2}$, the DomanyKinzel model reduces to directed bond percolation. This model is known to exhibit a continuous phase transition belonging to the universality class of directed percolation at the critical point $p_{c}=0.6447001(2)$ if the system size is infinite. In fact, as shown in Fig. 6, the transformation (6) maps an apparently disordered time series into an ordered one, where a typical DP cluster can be seen.

It should be stressed that in the present model the corresponding DP process takes place on a finite lattice so that for any finite $n$ there is no phase transition in a strict sense. Nevertheless, it is possible to observe the typical signatures of DP critical behavior within a certain temporal range which grows with $n$, as will be shown in the following.

\section{A. Two-point correlation function}

In order to see a signature of DP critical behavior we tried to identify the critical exponents in $1+1$ dimensions:

$$
\beta=0.276486(8), \nu_{\perp}=1.098654(4), \nu_{\|}=1.733847(6) \text {. }
$$

To this end we iterated the time series slightly above the critical point $p=p_{c}+0.001=0.6448$ and measured the connected part of the two-point correlation function

$$
c(\tau)=\left\langle x_{t} x_{t-\tau}\right\rangle-\left\langle x_{t}\right\rangle^{2} .
$$

Before the average was taken, the time series was equilibrated over $2 \times 10^{9}$ iterations in order to reach a stationary 


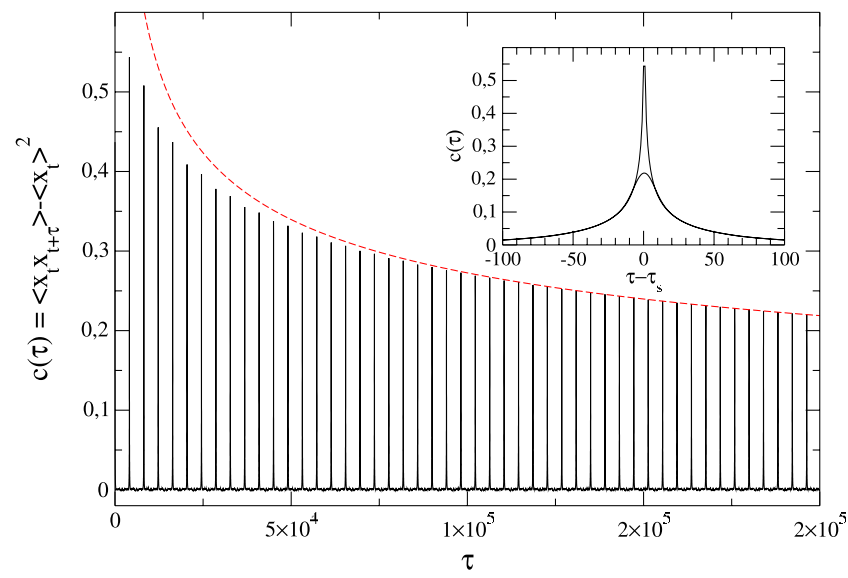

FIG. 7. (Color online) Two-point correlation function of the time series $c(r)$ defined in Eq. (14). The dashed line demonstrates that the spike peaks decay as $\tau_{s}^{-2 \beta / \nu_{\|}}$. The inset shows a zoom of the first and the last spikes.

state. Moreover, we chose a very large delay $n=4096$ to prevent the system from entering the zero sequence due to finite-size effects. Figure 7 shows a train of spikes at regularly spaced times $\tau_{s}$ which are caused by correlations between subsequent rows in the two-dimensional representation. The asymptotic envelope of these spikes seems to obey an asymptotic power law

$$
c\left(\tau_{s}\right) \sim \tau_{s}^{-\gamma}
$$

with an exponent $\gamma=0.32(4)$. The relation to DP predicts this exponent to be given by

$$
\gamma=2 \beta / \nu_{\|} \approx 0.318
$$

A similar attempt to obtain the spatial correlation exponent $2 \beta / \nu_{\perp}$ from the form of a single spike (see inset) fails. This can be explained as follows. In the central panel of Fig. 6 the form of the spike would correspond to a correlation function in the horizontal direction, whereas in the symmetrized representation shown in the right panel this correlation function would be tilted. Therefore, the spike profile is given by an interplay of both exponents $\nu_{\|}$and $\nu_{\perp}$, making it difficult to distinguish between them.

\section{B. Dynamical scaling of the pair connectedness function}

In order to identify the DP critical exponents more clearly, we measured the counterpart of the so-called pair connectedness function (see, e.g., [16]) at criticality $p=p_{c}$. The iteration starts with a single active seed $x_{n}=\delta_{n, n_{0}}$. After reordering the time series and representing it in the right panel of Fig. 6, we measure the density $\rho(\Delta, y)$ at a vertical distance $y$ and horizontal distance $\Delta$ from the seed. Since the pair connectedness function is known to obey the scaling form

$$
\rho(\Delta, y)=\Delta^{-2 \beta / \nu_{\perp}} h\left(y / \Delta^{1 / z}\right)
$$

with a universal scaling function $h$, the exponents can be determined by plotting $\rho(\Delta, y) \Delta^{2 \beta / \nu_{\perp}}$ versus $y / \Delta^{1 / z}$ in such a way that data sets for different values of $\Delta$ collapse. Plug-

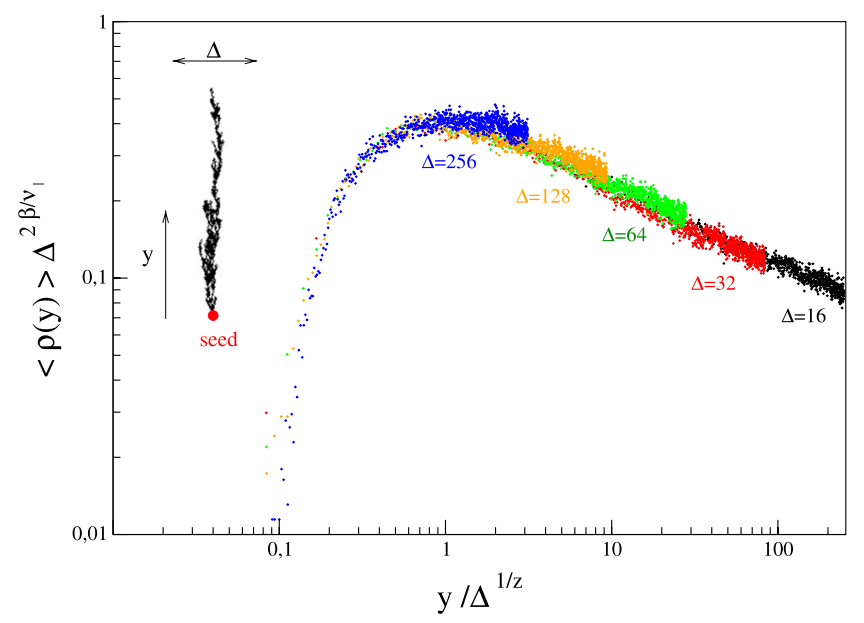

FIG. 8. (Color online) Data collapse for the counterpart of the pair connectedness function for $n=512$ and $k=1$.

ging in the known exponents of DP, one obtains a convincing data collapse, as shown in Fig. 8. This confirms unambiguously the critical behavior we expected to get.

\section{DISCUSSION}

In this paper, we have shown how a time series evolving according to a nonlocal update rule can be mapped onto a local process in two dimensions with special time-delayed boundary conditions. One interesting question is whether this result holds also for the continuous case. For example, discretizing a differential equation of the form

$$
\dot{x}(t)=F[x(t)]+G[x(t-\tau)]
$$

with delay $\tau$ and arbitrary functions $F$ and $G$, one gets

$$
x_{m}-x_{m-1}=h\left[F\left(x_{m}\right)+G\left(x_{m-k}\right)\right],
$$

where $h$ is the step size and $k=\tau / h$ is the discrete analog of the delay. In the present paper we have shown that the equation

$$
x_{m}-x_{m-s}=h\left[F\left(x_{m}\right)+G\left(x_{m-k}\right)\right]
$$

with $1<k<s$ exhibits (up to boundary conditions) the same properties as long as $s$ and $k$ are coprime. The question is whether it is possible to find an appropriate limit of (20) and recover (18). Work in this direction is currently under way [19].

\section{ACKNOWLEDGMENTS}

This work was motivated by the research of M. Ackermann, who studied a delayed time series with an update rule related to directed percolation. S.R.D. would like to thank the Alexander von Humboldt Foundation for financial support and the University of Würzburg for hospitality.

\section{APPENDIX: PROOF OF THE TRANSFORMATION}

The transformation (6) is proven in two steps. First we show that two sites of the time series separated by a time 
delay of $\Delta t=n$ are still separated by the same delay after the transformation. Then we show that sites that were originally separated by a time delay $\Delta t=k$ are mapped onto new sites with a time delay of either $\Delta t=1$ or $\Delta t=n+1$.

We start with the sites separated by $n$ time steps, that is, $t$ and $t-n$. According to the transformation rule we get

$$
\begin{aligned}
T(t)-T(t-n)= & (t q) \bmod n+n\lfloor t\rfloor_{n} \\
& -\underbrace{(t-n) q \bmod n}_{=(t q) \bmod n}-n\lfloor(t-n)\rfloor_{n} \\
& =n\left[\lfloor t\rfloor_{n}-\lfloor(t-n)\rfloor_{n}\right]=n,
\end{aligned}
$$

where we used the fact that for two sites of different blocks (which is always the case here) one has $\lfloor t\rfloor_{n}-\lfloor(t-n)\rfloor_{n}=1$.

Next we consider the case where sites have a delay of $k$, i.e.,

$$
\begin{aligned}
T(t)-T(t-k)= & (t q) \bmod n-[(t-k) q] \bmod n \\
& +n\left(\lfloor t\rfloor_{n}-\lfloor t-1\rfloor_{n}\right) .
\end{aligned}
$$

Here we have to distinguish two cases. If $t$ is a multiple of $n$ this expression reduces to

$$
T(t)-T(t-k)=-\underbrace{(-k q) \bmod n}_{=n-1}+n(\underbrace{\lfloor t\rfloor_{n}-\lfloor t-1\rfloor_{n}}_{=1})=1 .
$$

On the other hand, if $t$ is not a multiple of $n$ we get

$$
\begin{aligned}
T(t)-T(t-k) & =(t q) \bmod n-(t q-k q) \bmod n+n\left(\lfloor t\rfloor_{n}-\lfloor t-1\rfloor_{n}\right) \\
& =\underbrace{(t q) \bmod n}_{\in\{1, \ldots, n-1\}}-(\underbrace{(t q) \bmod n}_{\in\{1, \ldots, n-1\}}-\underbrace{(k q) \bmod n}_{=1}) \bmod n+n\left(\lfloor t\rfloor_{n}-\lfloor t-1\rfloor_{n}\right) \\
& =1+n\left(\lfloor t\rfloor_{n}-\lfloor t-1\rfloor_{n}\right)= \begin{cases}1 & \text { if } t \bmod n \geq k, \\
n+1 & \text { if } t \bmod n<k .\end{cases}
\end{aligned}
$$

[1] S. H. Strogatz, Nature (London) 394, 316 (1998).

[2] A. Cho, Science 314, 37 (2006).

[3] S. Boccaletti et al., Phys. Rep. 329, 103 (2000).

[4] A. Pikovsky, M. Rosenblum, and J. Kurths, Synchronization: A Universal Concept in Nonlinear Science (Cambridge University Press, Cambridge, U.K., 2001).

[5] I. G. Szendro and J. M. López, Phys. Rev. E 71, 055203(R) (2005).

[6] A. Argyris et al., Nature (London) 437, 343 (2005).

[7] H. J. Wünsche et al., Phys. Rev. Lett. 94, 163901 (2005).

[8] I. Kanter, N. Gross, E. Klein, E. Kopelowitz, P. Yoskovits, L. Khaykovich, W. Kinzel, and M. Rosenbluh, Phys. Rev. Lett. 98, 154101 (2007).

[9] J. K. Hale, Introduction to Functional Differential Equations (Springer, New York, 1993).
[10] A. Amann, E. Schoell, and W. Just, Physica A 373, 191 (2007).

[11] L. S. Tsimring and A. Pikovsky, Phys. Rev. Lett. 87, 250602 (2001).

[12] D. Bratsun, D. Volfson, L. S. Tsimring, and J. Hasty, Proc. Natl. Acad. Sci. U.S.A. 102, 14593 (2005).

[13] G. Giacomelli and A. Politi, Phys. Rev. Lett. 76, 2686 (1996).

[14] E. Domany and W. Kinzel, Phys. Rev. Lett. 53, 311 (1984).

[15] W. Kinzel, Z. Phys. B: Condens. Matter 58, 229 (1985).

[16] H. Hinrichsen, Adv. Phys. 49, 815 (2000).

[17] G. Ódor, Rev. Mod. Phys. 76, 663 (2004).

[18] S. Lübeck, Int. J. Mod. Phys. B 18, 3977 (2004).

[19] S. R. Dahmen and H. Hinrichsen, e-print arXiv:cond-mat/ 0703301. 